\section{PTU-099 EVALUATION TO SUPPORT SERVICE IMPROVEMENT FOR PATIENTS WITH INFLAMMATORY BOWEL DISEASE AT ST MARK'S HOSPITAL}

Ailsa Hart*, Marian O'Connor. St Mark's Hospital, London North West University Healthcare NHS Trust, London, UK

\subsection{6/gutjnl-2018-BSGAbstracts.477}

Introduction As a centre for excellence for Inflammatory Bowel Disease (IBD), St Mark's Hospital in North-West London attracts worldwide referrals while also treating local patients. As demand increases, yet NHS resource remains limited, pressure has heightened on the IBD service to improve capacity and reduce waiting times. This local service evaluation (SE) aimed to describe the current management of IBD outpatients, and patient and staff experience with the service, so that barriers to efficient care delivery could be identified and addressed.

Methods A SE involving the collection of retrospective and prospective clinic and patient level data was conducted in 2015 for patients attending outpatient clinics over one year. Data collected included numbers of patients attending clinics, disease characteristics, type of healthcare professional (HCP) seen and time from decision to prescribe a biologic to first dose $(n=31)$. Surveys were conducted with patients with IBD $(n=66)$ and HCPs $(n=46)$. Key personnel were interviewed in 2017 to determine how results could be used to drive specific service changes.

Results A mean of 18 patients were seen per clinic (with a maximum of 41 patients); $73 \%$ patients were in follow-up and attending for routine review. Of 51 patients followed prospectively, $8 \%$ had a joint visit involving multiple HCPs and $59 \%$ were judged to be in remission by the care team. Median time from decision to prescribe biologic therapy to first dose was 13.6 weeks. While $80 \%$ patients reported clinic management was 'good'/'very good', patients and HCPs were concerned about clinic space, patient volume and waiting times. Following the SE, a more integrated approach to treating patients and allocating staff resource was implemented to improve the pathway of care for patients (e.g. joint IBD multi-disciplinary clinics).

Conclusions of the high number of patients attending clinics, many had stable disease or were in follow-up. Many patients were reviewed by different HCPs on different days, driving a plan for joint clinics to improve the patient journey. There were delays accessing parts of the service (e.g. biologic therapy). Patients and staff reported dissatisfaction with clinic space and capacity. These findings helped generate an action plan for specific service-level changes such as specialist IBD/ joint multidisciplinary clinics, new administrative processes, and facilitation of patient self-management. This project can be used as a model for others to identify and address barriers to quality and efficiency within their own services.

\section{PTU-100 INPATIENT ENDOSCOPY: A 'HOT SITE' EXPERIENCE}

Mohamed Hussein*, Ezgi Ozcan, Mehul Patel, Kwok Tang, Akeel Alisa, Saif Musa. Royal Free Hospital NHS Trust, London, UK

\subsection{6/gutjnl-2018-BSGAbstracts.478}

Introduction Access to inpatient endoscopy is important for both patient management and flow within acute NHS Trusts. A daily dedicated weekday inpatient endoscopy list was introduced at Barnet Hospital, Royal Free London NHS Trust, to enhance service provision in line with NICE guidance (CG141, 2016). We report on our initial 'hot site' experience.

Method A single centre retrospective study involving of all consecutive inpatients requiring endoscopy at a large district general hospital (445 beds) serving a population 500000 during a 5 month period (January - May 2016). All patients were identified from the endoscopy procedure log. Additional data including endoscopy type, indication, therapeutic intervention and hospital discharge within 24 hours of endoscopy were collected using electronic patient records and the 'Unisoft GI Reporting Tool'.

Results In total 440 inpatient endoscopies were performed; $322(73 \%)$ gastroscopies, 82 (19\%) flexible sigmoidoscopies and $36(8 \%)$ colonoscopies. Median age was 76 years [interquartile range (IQR) 55-86], 53\% were male. Gastrointestinal bleeds (GIB) accounted for 192/440 (44\%) procedures. 40/192 (21\%) lower gastrointestinal and 152/192 (79\%) upper gastrointestinal bleeds (UGIB). Additional indications included 48/ 440 (11\%) abnormal imaging, 48/440 (11\%) dysphagia, 47/ $440(11 \%)$ iron deficiency anaemia, 40/440 (9\%) diarrhoea, 26/440 (6\%) percutaneous endoscopic gastrostomy (PEG) tube insertion, $17 / 440$ (4\%) abdominal pain, 17/440 (4\%) weight loss, 4/440 (1\%) volvulus and $1 / 440(<1 \%)$ for dyspepsia. Sedation was used in 315/440 (72\%) cases, median midazolam dose was $2.5 \mathrm{mg}$ [IQR $1-5 \mathrm{mg}$ ] and fentanyl $25 \mathrm{mcg}$ (IQR 0 $50 \mathrm{mcg}$ ). Median (IQR) procedure time for gastroscopy, flexible sigmoidoscopy and colonoscopy were 20 (IQR 15-25), 15 (IQR 10-20) and 30 (IQR 25-40) min, respectively. Colonoscopy completion rate was $31 / 36(86 \%)$. Therapeutic intervention occurred in $88 / 440$ (20\%). In total $259 / 440$ (59\%) procedures were undertaken within 24 hours of request, 110/ 152 (73\%) UGIB and 30/40 (75\%) lower GI bleeds. Overall 30 day mortality was $47 / 440 \quad(11 \%)$ and $114 / 440$ (26\%) patients were discharged within 24 hours.

Conclusion Gastrointestinal bleeding is the most common indication for inpatient endoscopy. A dedicated inpatient endoscopy list improves both patient management and flow with $>70 \%$ GIB scoped within 24 hours and a quarter of all patients discharged within 24 hour. Therapeutic procedures are expected in a fifth of all inpatient endoscopy. Optimal list timing merits further exploration, as does the prospect of extending the service to further improve outcomes.

\section{PTU-101 FIRST DEMONSTRATION OF TRAINEE-LED NETWORKS DELIVERING QUALITY IMPROVEMENTS IN GASTROENTEROLOGY SERVICES: A CALL TO ACTION}

The GARNet, Richard Ingram*. Gastroenterology Audit and Research Network, East Midlands

10.1136/gutjnl-2018-BSGAbstracts.479

Introduction The GARNet was the first trainee-led gastroenterology network to complete a multi-site audit. We focussed on standards of care and outcomes in acute upper GI bleeding (AUGIB). Here, we present our regional experience with quality improvement (QI) and our subsequent re-audit.

Methods We audited patient care against national standards (NICE CG141 and QS38). Patients aged $\geq 16$ years admitted with suspected AUGIB who underwent an inpatient OGD were prospectively identified between $01-30 / 11 / 16$ and $01-30 / 11 / 17$. QI focused on reducing time from presentation to endoscopy, using process mapping and staff questionnaires, to develop local action plans at each site. Fishers, Mann-Whitney and Wilcoxon 
tests were used for categorical, unpaired and paired continuous variables respectively.

Results See Table 1. 7 sites were able to participate in both rounds. There was a significant increase in the documentation of GBS and rebleed plans (vs. audit standard of 100\%). There were non-significant reductions in the median time to OGD and the proportion within 24 hour. This improved at 5 sites ( $p>0.05$ in paired analysis), and 5 vs. 6 sites achieved JAG $50 \%$ standard (no sites achieved 75\% standard). In the 2017 cohort, $42 \%$ of patients had OGD delayed $>24$ hour. They had significantly lower GBS and longer length of stay (see Table 2). In patients receiving endotherapy, OGDs were more timely (18.4 hour [11.3-25.9] vs. 22.8 hour [16.9-43.5], $\mathrm{p}=0.005$ ) but $31 \%$ were still treated after 24 hour.

Conclusions Locally-tailored QI driven through regional traineeled audit can deliver modest improvements in patient care. This audit shows that further action is needed to meet standards. Time to OGD is a pragmatic measure of quality of care and not a clinical outcome. Service development would benefit greatly from a tool to identify patients most likely to benefit from timely endoscopic diagnosis and endotherapy. We propose that QI is co-ordinated at national level. We are collaborating with our fellow trainee networks to support such initiatives.

\begin{tabular}{llll} 
Abstract PTU-101 Table 1 & & \\
\hline Patients (n) & 166 & 154 & \\
\hline Sites (n) & 9 & 8 & \\
Age (years, mean \pm SD) & $67 \pm 17.9$ & $67 \pm 18.7$ & \\
Sex (\% male) & 60 & 58 & \\
PPI pre-OGD (PPI-naïv [\%]) & $50 / 106[47 \%]$ & $41 / 87[47 \%]$ & ns \\
GBS recorded pre-OGD (\%) & 42 & 57 & 0.010 \\
Time to OGD (h, median [IQR]) & $23.0[16.5-$ & $22.0[15.1-$ & ns \\
OGD within 24 hour (\%, [range & $40.1]$ & $32.2]$ & \\
sites]) & $52[28-71]$ & $58[22-70]$ & ns \\
Rebleed plan [\%] & & & \\
\hline
\end{tabular}

\section{Abstract PTU-101 Table 2}

\begin{tabular}{llll}
\hline Patients (n) & 89 & 65 & \\
\hline Median GBS [IQR] & $10[5-13]$ & $8[5-11]$ & 0.048 \\
GBS $\geq 8(\%)$ & 69 & 51 & 0.030 \\
GBS $\geq 12(\%)$ & 38 & 20 & 0.021 \\
Shock index [IQR] & $0.78[0.61-$ & $0.73[0.60-$ & $\mathrm{ns}$ \\
& $0.94]$ & $0.89]$ & \\
Shock index $\geq 1(\%)$ & 18 & 11 & $\mathrm{~ns}$ \\
Endotherapy (\%) [number variceal band ligation, & $33[8-21]$ & $20[3-10]$ & $\mathrm{ns}$ \\
non-variceal] & & & \\
Length of stay (d) [IQR] & $5[3-8]$ & $6[5-10]$ & 0.017 \\
\hline
\end{tabular}

GBS, Glasgow Blatchford score; $n s$, not significant (2-tailed $\mathrm{p} \geq 0.05$ ); PPI, proton pump inhibitor
Introduction GI bleeding is a common emergency. NCEPOD 2015 guidance recommends Consultant input within 1 hour, to both major upper and lower GIB; performance of endoscopy within 24 hours for all patients, and within 2 hours of stability in haemodynamically unstable patients. This guidance was incorporated into our local Trust GI bleed pathway. We have a 24/7 GIB on call rota and inpatient endoscopy lists 6 days/week. We have previously used our electronic endoscopy requesting system to identify the source of any delays in performing endoscopy. Following this we modified vetting and booking practices. We aimed to audit compliance with NCEPOD guidance and against our previous audits.

Methods Data were prospectively collated over 4 weeks from $6 / 2 / 17$ to $5 / 3 / 17$. Major GI bleeds were identified with either Glasgow Blatchford Score (GBS) score $>8$ or pre-endoscopy Rockall $>5$ or Shock index $>1$. Information was extracted from the hospital electronic health record and the Unisoft endoscopy reporting tool.

Results 42 patients presented with upper GIB (UGIB) and 9 with lower GIB (LGIB). 95\% of patients with UGIB had a pre-endoscopy Rockall score documented, compared to $83.3 \%$ post endoscopy. Shock index was documented in $82.3 \%$ of all GIB patients.

$66.7 \%$ of patients with LGIB had a PR exam and only $33.3 \%$ also underwent proctoscopy. $66.7 \%$ of patients had a flexible sigmoidoscopy and $33.3 \%$ had a colonoscopy.

\section{Abstract PTU-102 Table 1}

\begin{tabular}{ll}
\hline Admission - medical review & 3 hours $45 \mathrm{mins}$ \\
\hline Medical review - endoscopy request & 4 hours $18 \mathrm{mins}$ \\
Endoscopy request - vetting & 10 hours $39 \mathrm{mins}$ \\
Vetting - endoscopy & 21 hours $45 \mathrm{mins}$ \\
\hline
\end{tabular}

$15 / 51$ of patients presented with major GIB, all of which were upper GIBs. 10/15 of these patients were discussed with the on-call Gastroenterologist within 1 hour $(66.7 \%$ compliance). No patients presented with a major lower or upper GIB, with haemodynamic instability, during this time frame.

$80.4 \%$ underwent endoscopy within 24 hours. Table 1 demonstrates the breakdown of the mean cumulative length of time between each stage, from admission to undergoing endoscopy.

Conclusion Our audit demonstrates improved compliance with NCEPOD and NICE guidance. Following changes to the endoscopy requesting process, the $\%$ of patients undergoing endoscopy within 24 hours has improved (26\% vs $80.4 \%$ in this re-audit). However, the incorporation of LGIB into the pathway revealed poor rates of compliance with proctoscopy and PR examination. Better communication is necessary between the surgical and medical teams for patients presenting with LGIB. Rapid Consultant Gastroenterologist input to patients with a major GIB happens frequently but needs further improvement. The introduction of an on call Gastroenterology baton telephone, and junior doctor education have been used to support the on call medical registrar with this.

\section{PTU-103 COMPARATIVE RE-AUDIT OF NOVEL BLEEDS ROTA PROVISION IN A LARGE DISTRICT GENERAL HOSPITAL}

Nasir Javed*, Ahmad Shirazi-Nejad, Hafiz Gulzeb Kamil, Anurag Agrawal. Doncaster Royal Infirmary, Doncaster, UK
Maryam Jan*, Paul Bumford, Adrian Thuraisingam. Wirral University Teaching Hospitals, Wirral, UK 